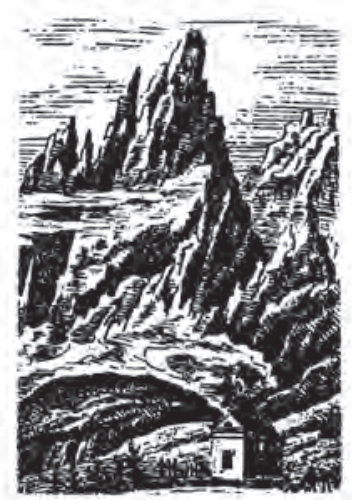

Corinne Geering

ORCID: 0000-0002-2334-0345

Leibniz-Institut für Geschichte und Kultur des östlichen Europa (GWZO)

corinne.geering@leibniz-gwzo.de

https://doi.org/10.19195/2084-4107.13.19

\title{
Zufluchtsorte in den Bergen: Die Bautätigkeit von Gebirgsvereinen und die Idealisierung der Karpaten in der Moderne
}

Słowa-klucze: Karpaty, architektura, rozwój przestrzenny, towarzystwa górskie, turystyka Keywords: Carpathians, architecture, spatial development, mountain clubs, tourism

Finding shelter in the mountains: The construction activity of mountain clubs and the idealisation of the Carpathians in the modern period

Summary

Mountainous regions have long been considered dangerous and difficult to penetrate. Only few people used to enter the widely uninhabited landscape for occupational purposes such as herding cattle, transporting goods, and mapping and surveying the land. At night and in the case of bad weather, these people found refuge at higher altitudes in rudimentary mountain huts. In the 
nineteenth century, the number of mountain travellers increased rapidly due to the construction of new train lines and other means of transportation, and they set out to spend their leisure time with climbing and hiking. This novel recreational use of mountain landscapes placed higher demands on local facilities and infrastructure. Newly established alpine clubs attended to the construction of new mountain huts from the Alps through the Carpathians to the Caucasus. This article discusses the construction activity of alpine clubs as a process of idealisation that continues to shape mountain landscapes until today.

Idealisation was not only achieved by means of written and visual representation, but complementarily by means of infrastructure, and it thus had a strong impact on the local social fabric. Mountain huts played a pioneering role in the touristic development of mountain regions, and in many instances, well equipped guesthouses and hotels were later constructed at the same site. This article analyses the appropriation of mountain landscapes through mountain huts and pays particular attention to the interactions between members of alpine clubs and the local population living in the mountains. The discussion is based on a set of travelogues, guidebooks, and annals by the Tatra Society, the Hungarian Carpathian Society, and the Transylvanian Carpathian Society which were published between the foundation of the first alpine clubs in the Carpathians in the 1870s and the beginning of the First World War. By highlighting the role of social background of agents, this article seeks to go beyond the focus in scholarship on nationalist interpretations. Rather, it reveals how landscape architecture attributed new cultural values to mountains in modernity.

Die physische Form von Landschaften ist untrennbar verbunden mit ihrer Darstellung und Interpretation. ${ }^{1}$ Berglandschaften sind davon nicht ausgenommen. In der Moderne hat sich deren Wahrnehmung und Gestalt wesentlich verändert und an die Stelle von Furcht ist Faszination getreten. Berge boten den Bergsteigenden und -reisenden Projektionsflächen für ihre eigenen Erfahrungen und kulturellen Prägungen. ${ }^{2}$ Diese bildhafte Dimension der Landschaft ist das Resultat eines bewussten Prozesses, in dem soziale Ungleichheit und Unebenheiten in der natürlichen Umwelt harmonisiert wurden. ${ }^{3}$ Die Landschaft fungierte als Mittel für die Herstellung sozialer Ordnung ${ }^{4}$ und die Idealisierung der Berge fand nicht nur in schriftlichen und bildlichen Darstellungen auf der Ebene der Interpretation statt, sondern komplementär durch aktive Nutzung und bewusste Gestaltung des Gebirgsraums. Vor diesem Hintergrund entwirft dieser Artikel eine sozialhistorische Perspektive auf die Bautätigkeit von Gebirgsvereinen in den Karpaten in der Moderne, die im Hinblick auf die sozioökomischen Unterschiede der Akteure über die in der Forschungsliteratur vorherrschenden nationalen Deutungen hinausgeht.

Im Fokus dieses Artikels stehen Schutzhütten, die für Menschen in einem ungangbaren und infrastrukturell anderweitig nicht erschlossenen Gebiet Schutz vor Unwetter und einen Zufluchtsort zur Rast oder Übernachtung boten. Alternativ auch Schutzhaus, Berghütte oder Bergbaude genannt, unterscheidet sich die horská chata,

${ }^{1}$ Die Definition von Landschaft und der Zusammenhang zwischen ihrer physischen Realität und der von Menschen hervorgebrachten Repräsentation sind intensiv diskutierte Fragen der Geographie. Einen guten Überblick bietet Karen M. Morin 2009, S. 286-299.

2 So beispielsweise der britische Schriftsteller Robert MacFarlane (2003) in seiner autobiographisch geprägten Geschichte des Bergsteigens.

3 Siehe Cosgrove 2006, S. 51.

${ }^{4}$ Siehe Mitchell 2002. 
útulňa, menedékház oder schronisko von ländlichen Wochenendhäusern und Herbergen, wie sie im Verlauf des 20. Jahrhunderts an Bedeutung gewannen. ${ }^{5}$ Schutzhütten wurden zunächst von Grundbesitzern, unternehmerisch tätigen Privatpersonen in Gastronomie und Tourismus sowie von staatlichen Behörden in der Forstwirtschaft und der Grenzsicherung errichtet. Mit dem Aufkommen des Tourismus in der zweiten Hälfte des 19. Jahrhunderts nahmen sich von Europa über Nordamerika bis Australien nun auch neu gegründete Gebirgsvereine der Bautätigkeit an. Mehrere historische Arbeiten haben sich mit Gebirgsvereinen befasst, indem sie zumeist auf einzelne Vereine oder Regionen fokussierten. ${ }^{6}$ Im Gegensatz dazu arbeitet dieser Artikel synthetisch mit Beispielen aus dem gesamten Karpatenraum, indem er anhand der Errichtung und Nutzung von Schutzhütten die Aneignung des Gebirgsraums und die damit verbundene Interaktion mit der lokalen Bevölkerung als Idealisierungsprozess analysiert. Grundlage dafür stellen Reiseberichte, Reiseführer und die Vereinszeitschriften dar, die im Zeitraum von der Gründung der ersten Karpatenvereine in den 1870er Jahren bis zum Beginn des Ersten Weltkriegs erschienen. Dazu gehören das deutsch- und ungarischsprachige Jahrbuch des Ungarischen Karpathen-Vereines/Magyarországi Kárpátegylet évkönyve ${ }^{7}$, das deutschsprachige Jahrbuch des Siebenbürgischen Karpathen-Vereins sowie das polnischsprachige Jahrbuch der Tatra-Gesellschaft (Pamiętnik Towarzystwa Tatrzańskiego). ${ }^{8}$ Obschon diese von Reisenden verfassten Quellen eine Perspektive von außen auf den Gebirgsraum einnehmen, beinhalten sie vielfältige Begegnungen mit der Bergbevölkerung sowie mit Menschen, die sich aufgrund ihrer Arbeit als Jäger oder Gendarm dort aufhalten. In zwei Teilen folgt dieser Artikel den Begegnungen im vermeintlich leeren Gebirgsraum. Der erste Teil führt aus, welche Rolle die Schutzhütte in der Erschließung der Karpaten für den Tourismus spielte. Anschließend beleuchtet der zweite Teil die Raumaneignung durch die Gebirgsvereine und die damit verbundene Idealisierung der Berge.

\section{Die Schutzhütte verschiebt die Grenze des Bekannten}

Die Karpaten sind ein über 1500 Kilometer langes Gebirge, das im hier untersuchten Zeitraum zu großen Teilen in Österreich-Ungarn und den angrenzenden Gebieten lag. Der Gebirgszug erstreckt sich mit seinen Ausläufern von den Beskiden (Beskydy) im östlichen Mähren über die Tatra an der damals galizisch-ungarischen

${ }^{5}$ Neben von den Gebirgsvereinen selbst herausgegebenen Publikationen bieten sozial- und architekturhistorische Arbeiten einen Überblick: Grupp 2003, S. 172-181; Kulig 2003; Kerschbaumer 2017. Zur Abgrenzung von ländlichen Wochenendhäusern siehe: Schüler 1937.

6 Polášková/Polášek 2009; Kurek/Wójcik (Hrsg.) 2016; Wedekind 2004, S. 71-86.

${ }^{7}$ Das Jahrbuch wurde 1874-1883 zweisprachig auf Deutsch und Ungarisch herausgegeben, ab 1884 in sprachlich getrennten Ausgaben.

8 Aufgrund der Mehrsprachigkeit der Karpatenregion verwendet dieser Artikel bei der ersten Erwähnung von Ortschaften die Namen in mehreren Sprachen und anschließend nur die deutsche Variante. Bei Bezeichnungen von Vereinen, Schutzhütten, Seen und Tälern beschränkt er sich auf Deutsch mit der anderssprachlichen Variante aus der Quelle in Klammern. 
Grenze bis nach Siebenbürgen und dem Banat im Süden, einschließlich des damaligen ungarisch-rumänischen Grenzgebietes. Das Interesse an den Karpaten bei ihrer Erschließung seit dem 18. Jahrhundert galt im Vergleich zu den Alpen weniger der Verbindung von Verkehrsachsen, sondern vor allem der geopolitischen Relevanz des Gebirgszuges. ${ }^{9}$ Grenzübergänge spielten auch in den Reiseberichten eine wichtige Rolle, indem Reisende immer wieder auf die Gendarmerie und Zollposten trafen, die ihnen manchmal auch Unterkunft boten. Gleichzeitig bedeuteten die Grenzverläufe eine Einschränkung der touristischen Bewegungsfreiheit. Dies stieß auch auf Kritik: So wünschte sich ein Wanderer im ungarisch-rumänischen Grenzgebiet, dass er in den Bergen nicht seinen Pass, sondern seine Mitgliedskarte des touristischen Vereins vorweisen könne. ${ }^{10}$ Dass auch touristische Vereine nicht vor Kontroversen um staatliche Grenzverläufe gefeit waren, zeigt der jahrzehntelange Rechtsstreit um die staatliche Zugehörigkeit des Sees Meerauge in der Hohen Tatra an der Grenze zwischen dem Kronland Galizien/Galicja/Halyčyna in Österreich und dem Komitat Zips/Szepes/Spiš in Ungarn. Der Streit, an dem sich auch die in der Region tätigen Gebirgsvereine und ihre Mitglieder beteiligten, wurde erst 1902 unter internationaler Rechtsprechung zugunsten Galiziens beigelegt. ${ }^{11}$

Vor dem Hintergrund solcher Auseinandersetzungen beschäftigt sich ein Großteil der Forschungsliteratur zur Erschließung und Aneignung des Gebirgsraums mit nationalen Deutungen der Landschaft. So wurde die Symbolik von Bergen, von den Alpen über den Balkan bis zu den Karpaten, von nationalen Projekten beansprucht und die Gebirgszüge figurieren bis heute prominent im Selbstverständnis verschiedener nationalstaatlicher Identitäten. ${ }^{12}$ Insbesondere aus Sicht der polnischen Nationalbewegung im späten 19. Jahrhundert nahmen die Karpaten, allen voran die Tatra, eine zentrale Rolle in der Konstruktion einer nationalen Landschaft ein. ${ }^{13}$ Neben der Landschaft war es auch die bislang weithin unbekannte lokale Bergbevölkerung, die Górale, die von Intellektuellen zum Inbegriff des polnischen Bauern erklärt wurden. ${ }^{14}$ Eine solche Vereinnahmung führte zu Konflikten zwischen Ansprüchen von verschiedenen Seiten, die sich in der Region über Jahrzehnte hinziehen konnten. ${ }^{15}$ Diese Konfliktlinien konnten sich innerhalb derselben nationalen Gemeinschaft zwischen Zentrum und Region auftun, wie Martin Pelc anhand der Beziehung der Pohorská jednota Radhošst (Gebirgsverein Radegast) und dem Klub českých turistů (Klub tschechischer Touristen) mit Sitz in Prag/Praha zeigt. ${ }^{16}$ Sie konnten sich aber auch anhand nationaler Zugehörigkeit ausgestalten, wie er in einem anderen Beitrag zum Riesengebirge

${ }^{9}$ Siehe Matuska 2012, S. 258-259; Heppner 2015.

10 Barcza 1904, S. 76.

11 Der Streit um das Meerauge. 1909. In: Österreichische Zeitschrift für Vermessungswesen 7.9, S. 279-281.

\footnotetext{
12 Lipták 2003, S. 261-288; Brunnbauer/Pichler 2002, S. 77-100; Zimmer 1998, S. 637-665.

13 Siehe Dabrowski 2005, S. 380-402; Hoenig 2014, S. 56-73.

14 Crowley 2001, S. 108.

15 Siehe Hoenig 2018.

16 Pelc 2008, S. 425-437.
} 
an der schlesisch-böhmischen Grenze argumentiert. So beschreibt er die dort im frühen 20. Jahrhundert neu errichteten Schutzhütten und Aussichtstürme als „nationale Bollwerke" tschechischer und deutscher Interessensvereinigungen. Die Berglandschaft sei „vollständig segregiert und einer konsequenten Ethnisierung unterzogen" worden und Verständigung zwischen den Parteien sei die Ausnahme gewesen. ${ }^{17}$ Im Gegensatz dazu führt Pieter Judson im Hinblick auf andere Regionen in Österreich-Ungarn an, dass für deutsche Reisende nationalistisch ausgerichtete Reiseführer und Unterkünfte vergleichsweise „eine nur sehr geringe Rolle“" gespielt hätten. ${ }^{18}$

$\mathrm{Ob}$ national oder anderweitig motiviert, stellte die Raumaneignung als Repräsentationsfläche für die eigenen Interessen eine wesentliche Motivation für die Errichtung von Schutzhütten in der Moderne dar. Von Reisenden wurde das Gebirge zunächst als unbewohnter und ungastlicher Raum beschrieben, vor dem sie erschraken. ${ }^{19}$ Die Karpaten waren im 19. und frühen 20. Jahrhundert für viele Reisende aus den Metropolen Österreich-Ungarns und dem westlichen Europa eine terra incognita, wie mehrere Quellen behaupteten. ${ }^{20}$ Die westlich zu den Karpaten gelegenen Alpen waren den Gebirgsreisenden häufig vertrauter. Für Patrice Dabrowski fungierten die Karpaten als eine europäische frontier, die am Rand eines Gebiets die Möglichkeit zu Begegnungen mit Unbekanntem bot. ${ }^{21}$ Von derselben expansionistischen Perspektive geht Marie Louise Pratts Konzept der contact zone aus, in der sich Akteure wie ein intellektueller Stadtbewohner und ein Berghirte begegnen, die vorab räumlich und historisch voneinander getrennt waren. Pratt legt den Fokus nicht auf den Unterschied zwischen Reisenden und Bereisten, sondern hebt in Reiseberichten ihre Interaktion hervor, die sich häufig in einem streng asymmetrischen Machtverhältnis abspielte. ${ }^{22}$

Wie die Alpen wurden die Karpaten im 19. Jahrhundert nicht auf Initiative der lokalen Bevölkerung für den Tourismus erschlossen, sondern von Alpinisten und Alpinistinnen aus den Städten Österreich-Ungarns oder aus anderen Ländern. Diese waren Teil einer international vernetzten Elite, die sich die Berge als ihr „Spielplatz Europas“ zu eigen machten, wie dies die berühmt gewordene Metapher des britischen Alpinisten Leslie Stephens ausdrückte. ${ }^{23}$ Einzelne Dörfer wie Zakopane wurden dabei zum Treffpunkt städtischer Intellektueller und stiegen innerhalb weniger Jahrzehnte zu einem touristischen Zentrum des geteilten Polens auf. ${ }^{24}$ Diese städtischen Bergreisenden waren jedoch zur Orientierung stets auf die Unterstützung der lokalen Bevölkerung angewiesen, die sie im unbekannten

17 Pelc 2014, S. 236, 241.

18 Judson 2014, S. 63.

19 Posewitz 1894, S. 14. Zur Rolle des Gebirgsraum ,jenseits der bewohnten Welt" siehe auch Scharfe 2007, S. 196-203.

20 Rey 1876 S. 7; Heksch 1885, S. vi; Scharr (Hrsg.) 2004.

21 Dabrowski 2008, S. 46.

22 Pratt 1992, S. 6-7.

${ }^{23}$ Stephen 1871.

24 Keym 2008. 
Gebirge führen konnte. In den Quellen fehlen aber häufig die Namen dieser Menschen, weshalb sie meistens in den Hintergrund der Analyse rücken. Zwei kürzlich erschienene kolonialgeschichtliche Publikationen nahmen sich den unterstützenden Begleitern und Begleiterinnen von europäischen Entdeckungsreisenden an und zeigten das große Potential einer solchen Forschungsperspektive auf. So machten diese Akteure im Hintergrund durch ihre Kenntnisse, Kontakte und nicht zuletzt durch ihre Muskelkraft die Reisen überhaupt erst möglich. ${ }^{25}$

Wie sich die Karpaten den Bergreisenden in der Mitte des 19. Jahrhunderts präsentierten, als noch kaum touristische Infrastruktur vorhanden war, gibt eine Lithographie von Carl Kořistka (Bild 1) aus dem Jahre 1864 wieder. Kořistka, der Professor am Polytechnischen Landesinstitut in Prag war, veröffentlichte seinen Reisebericht in die Hohe Tatra bei einem Verlag im Deutschen Reich. Seine Zeichnung zeigt den See Meerauge (Morskie Oko) ${ }^{26}$ im Fischbachtal (Dolina Rybiego Potoku), der bereits Anfang des 19. Jahrhunderts große Anziehungskraft für Reisende ausübte, wie Fremdenbücher aus den nahe gelegenen Dörfern belegen. ${ }^{27}$ In seinem Reisebericht beklagte sich Kořistka, dass bezüglich Unterkunftsmöglichkeiten in den Karpaten für „so gut wie gar nichts gesorgt“ sei. ${ }^{28}$ In den meisten Fällen müsse der Reisende froh sein, wenn er für die Nacht überhaupt ein dreckiges Bett in einem Kämmerchen eines Wirtshauses erhalten könne, das der Prager Professor aber insgesamt als zu teuer empfand. Eine bessere Unterkunft finde sich nur in den Kurorten, die jedoch für Gebirgstouren zu weit entfernt wären. Wie Kořistkas Wanderung prägten die fehlenden Unterkunftsmöglichkeiten auch die anderen Reiseberichte der Zeit, die sich entsprechend nur von Stadt zu Stadt bewegten und die Ausflüge in die Berge auf mehrstündige Wanderungen begrenzten. Erst in späteren Reiseberichten folgen mit Ausbau der Infrastruktur und der Errichtung von Schutzhütten mehrtätige Gebirgstouren mit Übernachtung.

Die ersten Berghütten wurden für Menschen errichtet, die in den Karpaten lebten und arbeiteten, damit sie beim Weiden oder beim Warentransport einen Ort zum Übernachten hatten. In den daneben bestehenden vereinzelten Wirtshäusern kehrten vornehmlich Waldarbeiter, Grenzwächter und Soldaten ein. Die damaligen Berghütten waren nicht sehr komfortabel eingerichtet und verbanden häufig Wohn- mit Stallraum. Ein Dach, das vor Regen, Schnee und Sturm schützte, und eine Feuerstelle, an der man sich wärmen oder auch etwas kochen konnte, mussten genügen. Solche Sennhütten kommen meistens dann in Reiseberichten vor, wenn sich die Reisenden verlaufen hatten oder von schlechtem Wetter überrascht wurden. Solange es in bestimmten Gegenden noch keine Hotels und Gasthäuser gab, waren die Reisenden auf langen Wanderungen auf die Gastfreundschaft der Bauern und Hirten angewiesen und bereiteten sich gar darauf vor, indem sie als

${ }^{25}$ Malzner/Peiter (Hrsg.) 2018; Volker 2018.

${ }^{26} \mathrm{Im}$ Deutschen damals auch als Fischsee bezeichnet, wie in der Bildunterschrift von Kořistkas Lithographie.

27 Weber 1897, S. 39.

${ }^{28}$ Kořistka 1864. 
zu entrichtende Gegenleistung Alkohol, Tabak oder andere Waren mittrugen. ${ }^{29}$ Auf einer Photographie der Wienerin Emma Lövy aus dem Jahre 1909 ist eine solche einfache Hütte zu sehen (Bild 2), die sich im Kar unterhalb der Piatra Rea im Rodnaer Gebirge befand und von einem jüdischen Hirten bewohnt war. Lövys Bericht ist zu entnehmen, dass sie die Begegnung mit den Hirten aktiv suchte. So schreibt sie, dass sie solche Möglichkeiten schätze, um die „ethnographischen und volkswirtschaftlichen Verhältnisse“ des Landes besser kennenzulernen. ${ }^{30}$

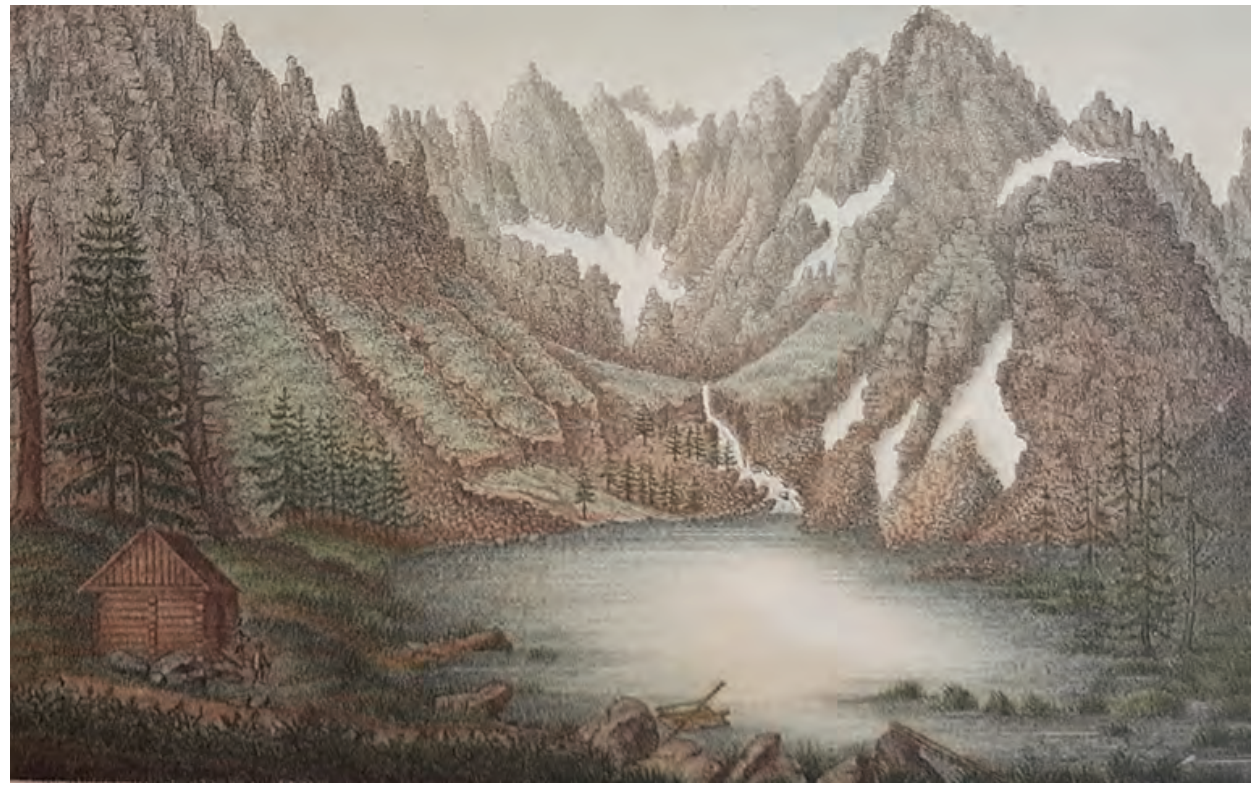

1. Carl Kořistka, 1864, Die Hohe Tatra in den Central-Karpaten. Eine geographische Skizze verfasst auf Grundlage einer Bereisung. Ergänzungsheft No. 12 zu Petermann’s „, Geographischen Mittheilungen“. Gotha: Justus Perthes. N. Pag. (C. Kořistka, Die Hohe Tatra in den CentralKarpaten. Eine geographische Skizze verfasst auf Grundlage einer Bereisung. Ergänzungsheft No.

12 zu Petermann’s „,Geographischen Mittheilungen“, Justus Perthes, Gotha 1864, [b.s.])

Mit der Zunahme der Anzahl der Reisenden im Gebirgsraum folgten den einfach gehaltenen Hütten solche mit höherem Ausbaustandard. Diese machten die Berge „für den Komfort und Bequemlichkeit schätzenden Naturfreund“ besser zugänglich, wie es ein Bericht aus dem Jahre 1898 treffend formulierte. ${ }^{31}$ Eine der ersten Hütten, die von Reisenden für ihre Freizeitaktivitäten benutzt wurde, ist auf der linken Seite der Lithographie von Kořistka am Ufer des Meerauges abgebildet. Diese Hütte wurde bereits im frühen 19. Jahrhundert zum ersten Mal erwähnt und im Auftrag des damaligen Grundbesitzers erbaut. In den Erinnerungen des

\footnotetext{
29 Kalchbrenner 1875, S. 237.

30 Lövy 1910, S. 17.

${ }^{31}$ L. 1898, S. 156.
} 
Malers und Tatrareisenden Walery Eljasz Radzikowski aus dem Jahre 1903 findet sich eine Beschreibung der Ausstattung dieser Hütte, die sich in einem desolaten Zustand befand. Das Dach fehlte, in der Mitte des Raums brannte ein Feuer und die Türen und Fensterläden waren bereits als Heizmaterial verbrannt worden. Da immer mehr Reisende in die Tatra kamen, empfand Eljasz Radzikowski das Fehlen einer angemessenen Unterkunft als Schande des polnischen Landesteils unter österreichischer Herrschaft (,wstyd dzielnicy Polski pozostającej pod rządem austryackim"), die erst durch die Gebirgsvereine abgewendet werden könnte. ${ }^{32}$ Noch heute steht dort die Meeraugehütte (Schronisko nad Morskim Okiem), die 1908 nach den Bränden der vorherigen Hütten von der Tatra-Gesellschaft errichtet wurde.

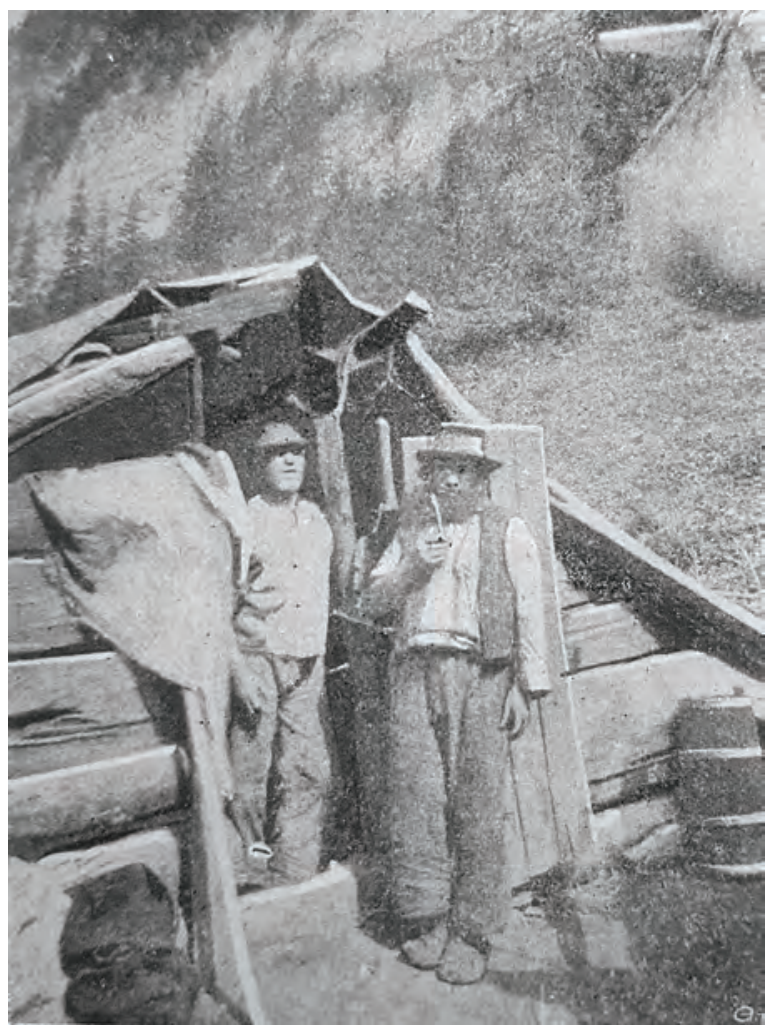

2. Emma Lövy, 1910, Ein Ausflug in die Rodnaer Alpen. In: Jahrbuch des Ungarischen Karpathenvereins 37, S. 29 (E. Lövy, Ein Ausflug in die Rodnaer Alpen, „Jahrbuch des Ungarischen Karpathenvereins” 37, 1910, s. 29)

32 Radzikowski 1903, S. 123. 


\section{Gebirgsvereine, Reisende und die Bergbevölkerung in der Kontaktzone}

Die Tatra-Gesellschaft (Towarzystwo Tatrzańskie) war nur einer der Gebirgsvereine, die in der zweiten Hälfte des 19. Jahrhunderts in den Karpaten gegründet wurden. Nach dem Österreichisch-Ungarischen Ausgleich 1867 stieg die Anzahl Vereine in beiden Reichshälften rasant an. ${ }^{33}$ Gebirgsvereine waren eine der Interessensvereinigungen, die sich der Erforschung und Gestaltung der umgebenden Landschaft widmeten. In vielen Ortschaften und Regionen Österreich-Ungarns bildeten sich Verschönerungs-, Touristen- und Wandervereine sowie Vereine zur Hebung des Fremdenverkehrs und zur Landeskunde. Sie alle koordinierten eine Vielzahl neuer Freizeitaktivitäten in der Berglandschaft. Während religiöse Pilgerfahrten zu Kirchen und Kapellen auf Höhenlagen eine längere Tradition hatten, stellten das Wandern, Bergsteigen und Skifahren neue Tätigkeiten dar, die bei manchen Zeitgenossen Verwunderung, wenn nicht gar Unverständnis oder Belustigung über diese strapazenreichen Hobbies auslösten. ${ }^{34}$

Nach der Gründung des Schweizer Alpen-Clubs (1863) und des Deutschen und Österreichischen Alpenvereins $(1862 / 1869)^{35}$ gab es auch erste Initiativen für einen ähnlichen Verein in den Karpaten. Dabei waren Mediziner, Armeeangehörige, Botaniker und Kunstschaffende federführend. Ein Aufruf für einen den gesamten Gebirgszug umfassenden Karpatenverein blieb jedoch ohne Resultat ${ }^{36}$ und kurz danach wurden 1873 die ersten regionalen Karpatenvereine gegründet: der Ungarische Karpathenverein/Magyarországi Kárpátegylet mit Sitz in Altschmecks/ Ótátrafüred/Starý Smokovec ${ }^{37}$, die Galizische Tatra-Gesellschaft (Galicyjskie Towarzystwo Tatrzańskie, bzw. Towarzystwo Tatrzańskie) in Krakau/Kraków und der Siebenbürgische Alpenverein ${ }^{38}$ in Kronstadt/Brașov/Brassó. Daneben

${ }^{33}$ In Österreich ermöglichte das Vereins- und Versammlungsrecht von 1867 die Gründung von Vereinen. In Ungarn war das Vereinsrecht bis zum Ende der Habsburgermonarchie nicht gesetzlich geregelt, sondern die Handhabung erfolgte über einen Erlass des Innenministeriums. Siehe auch: Toth 1990, S. 77.

34 Lüdecke 1884, S. 103.

35 Im Jahre 1873 zum Deutschen und Österreichischen Alpenverein zusammengefasst.

36 Gemäß der Darstellung des Militärarztes Theodor Posewitz (1894) zum Jubiläum des UKV ging die erste Initiative für einen Karpatenverein auf Heinrich Wallmann aus Salzburg zurück, der als k. u. k. Regimentsarzt in den 1860er Jahren zuerst in Kaschau/Košice/Kassa und anschließend in Lemberg/Lwów/L'viv stationiert war. Nach einer Wanderung in der Hohen Tatra habe dieser Aufrufe auf Ungarisch, Deutsch und Polnisch zur Gründung eines Kronländer und Komitate übergreifenden Karpatenvereins nach dem Vorbild des österreichischen Alpenvereins verfasst, den er in allen Landesteilen der Karpaten verteilen ließ.

37 Aufgrund des Wunsches einiger Mitglieder, den Vereinssitz nach Budapest zu verlegen, kam es 1892 zur Abspaltung von fünf Sektionen des Ungarischen Karpathenvereins, die sich neu zum Ungarischen Touristenverein (Magyar Turista Egyesület) mit Sitz in Budapest formierten.

${ }^{38}$ Der Siebenbürgische Alpenverein wurde später in den 1880 gegründeten Siebenbürgischen Karpathenverein eingegliedert. 
entstanden in den folgenden Jahrzehnten weitere kleinere regionale Vereine. ${ }^{39}$ Die Karpatenvereine waren wie die Vereine in den Alpen in regionale Sektionen unterteilt, die sich bestimmten Teilen des Berggebietes widmeten. Aufgrund der großen Anzahl deutschsprachiger Mitglieder des Ungarischen und Siebenbürgischen Karpathenvereins waren die Beziehungen zu den Alpenvereinen in Österreich und im Deutschen Reich intensiv. Im Gegensatz zur Tatra-Gesellschaft stellte die slawischsprachige Bevölkerung eine Minderheit in den ungarischen Vereinen dar. ${ }^{40}$ Durch Ehrenmitgliedschaften und Schriftentausch bestand von Beginn an eine Kooperation zwischen den Karpatenvereinen, auch über die ungarisch-galizische Grenze hinweg. ${ }^{41}$

Die Karpatenvereine hatten sich als Aufgabe die Erforschung und ErschlieBung des Gebirgsraums gesetzt. Deswegen verbreiteten sie Informationen für Reisende und versuchten ihre Bergregionen für den Tourismus zu popularisieren, indem sie eine eigene Vereinszeitschrift herausgaben, in naheliegenden Ortschaften Bergmuseen eröffneten, die lokale Hausindustrie förderten sowie photographische Aufnahmen, Landkarten und Reiseführer mit Angaben zu Kletter- und Wanderrouten veröffentlichten. Während es sich bei der Öffentlichkeitsarbeit in erster Linie um die Aufgaben der Vereinszentralen handelte, oblag die Gangbarmachung des Geländes vor allem den Sektionen. Diese waren auf die Zusammenarbeit mit den Gemeinden, den für die Forstwirtschaft zuständigen staatlichen Behörden, den Grundbesitzern und der lokalen Bevölkerung angewiesen. Die Aufgaben der Sektionen umfassten das Anlegen neuer Wege und das Anbringen von Markierungen, so dass sich die Reisenden im Gebirge zurechtfinden konnten. ${ }^{42}$ Ferner bildeten sie Männer aus der lokalen Bevölkerung zu Bergführern aus und installierten einen Bergrettungsdienst. So professionalisierten sie Dienstleistungen, die früher von ortsansässigen Förstern, Geistlichen und Notaren vermittelt worden waren. ${ }^{43}$

Zur Gangbarmachung des Geländes gehörte auch der Bau von Schutzhütten, wo Reisende auf längeren Routen eine Unterkunft finden konnten. Dem Bau von Schutzhütten gingen lange Vorbereitungs- und Sondierungsarbeiten und manchmal auch hitzige Debatten voraus, was die beste Platzierung der Hütte betraf. ${ }^{44}$ Finanziert

39 Dazu gehörten unter anderem der Mährisch-Schlesische Sudetengebirgsverein (1881), der Gebirgsverein Radegast/Pohorská jednota Radhošt' (1884), der Biharer Karpatenverein (1888), der Mecsek-Gebirgsverein (1891), der Beskidenverein (1893), und der Touristenverein jenseits der Donau/ Dunántuli Turista Egyesület (1903) in Sopron.

${ }^{40}$ Klipp schätzt die Anzahl slowakischer Mitgliedern auf etwa 10-15\%. Vgl. Klipp 2006, S. $177-178$.

${ }^{41}$ Siehe bspw. den Brief von 1875 von Egide Berzeviczy, dem Präsidenten des Ungarischen Karpathenvereins, in dem er sich für die Ehrenmitgliedschaft der Tatra-Gesellschaft' bedankt. Ważniejsze pisma wystosowane do Towarzystwa Tatrzańskiego. 1876. In: Pamiętnik Towarzystwa Tatrzańskiego 1, S. 36.

${ }^{42}$ Neben den Statuten der Gebirgsvereine bietet Dénes (1888) eine Zusammenfassung der Tätigkeiten.

43 Jahresberichte der Sektionen unseres Vereines. Sektion Broos. 1884. In: Jahrbuch des Siebenbürgischen Karpathen-Vereins 4, S. 150.

${ }^{44}$ Lüdecke 1884, S. 96. 
wurde der Bau und das Inventar der Schutzhütten sowie der aufwändige Transport des Materials teilweise durch die Mitgliederbeiträge des Vereins, meistens aber durch den Verkauf von Anteilscheinen, private Spenden oder Sponsoring und Kredite der lokalen Sparkassen und Kreditanstalten. Das Land und teilweise auch das für den Bau benötigte Holz wurde von den Grundbesitzern oder den staatlichen Behörden, allen voran dem Forstarär und dem Ackerbauministerium, häufig unentgeltlich zur Nutzung überlassen ${ }^{45}$ Im Gegenzug stellten die Gebirgsvereine ihre Schutzhütten auch Forstarbeitern zur Verfügung. Ähnliche Vereinbarungen zur gemeinsamen Nutzung der Infrastruktur wurden mit der staatlichen Zollinspektion getroffen. Die Sektion Hermannstadt des Siebenbürgischen Karpatenvereins beantragte 1885 die Mitbenutzung des Wachhauses und sah aufgrund der Zusage der Zollinspektion vom Bau einer eigenen Hütte $a b .{ }^{46}$

Über die Jahrzehnte hinweg wurden immer mehr Teile des Gebirges für Wanderungen und Bergsteigen mit Schutzhütten erschlossen. Der Ungarische Karpathenverein erbaute alleine in den ersten zehn Jahren zehn Hütten, der Siebenbürgische Karpathenverein bis zum Ersten Weltkrieg insgesamt 38 Schutzhütten und die Tatra-Gesellschaft in demselben Zeitraum elf Schutzhütten sowie sieben Gartenlauben..$^{47}$ Als Mitglied eines Gebirgsvereins hatte man Anspruch auf vergünstigte Übernachtungen in den Hütten des eigenen Vereins und der anderen Gebirgsvereine, mit denen entsprechende Abkommen geschlossen worden waren. Den Schlüssel erhielten die Reisenden üblicherweise bei einem Wirt oder bei anderen Bewohnern und Bewohnerinnen einer naheliegenden Ortschaft. Fehlte dieser Schlüssel, so konnte es durchaus vorkommen, dass die Reisenden versuchten, sich durch Einbruch anderweitig Zugang zur Hütte zu verschaffen. ${ }^{48}$

Die Vereinszeitschriften zeugen davon, dass sich die Mitglieder der Gebirgsvereine durchaus der Mehrsprachigkeit der Region und der Wirkung nationaler Benennungen ihrer Bauwerke bewusst waren. Dies zeigte sich besonders eindrücklich bei der Einweihung der Schutzhütte ,Schlesierhaus' am Felkersee (Velické pleso), die 1895 von der schlesischen Sektion des Ungarischen Karpathenvereins errichtet wurde (Bild 3). Bei der Feier betonten die anwesenden Mitglieder nationale Trennlinien, während sie diese gleichzeitig aufzuheben suchten. Witze über die Eroberung des Berggebiets durch Preußen wechselten sich mit dem folgenden Weihelied ab:

Wer hier ein Obdach fand, Reiche die Bruderhand

45 So tat dies beispielsweise Graf Teleki, der für den Bau einer Schutzhütte am Bulea-See der Sektion Hermannstadt des Siebenbürger Karpathenvereins sein Grundstück, Holz sowie seinen Förster zur Verfügung stellte. Siehe Lüdecke 1884, S. 96.

46 Jahresberichte der Sektionen unseres Vereins. Sektion Hermannstadt. 1886. In: Jahrbuch des Siebenbürgischen Karpathen-Vereins 6, S. 204.

47 Heltmann 1990, S. 11-26; Schroniska i altany Tow. Tatrz. w Tatrach. 1914. In: Pamiętnik Towarzystwa Tatrzańskiego 35, S. LLXVI-LXVII.

48 Siehe Magnesius 1897, S. 75. 
Dem Fremden gern!

Was drunten Völker trennt,

Droben kein Herz mehr kennt,

Jeder den Andern hier

Als Freund begrüsst. ${ }^{49}$

Die Betonung oder Ablehnung nationaler Zugehörigkeiten am Schlesierhaus verweisen darauf, dass sich die für touristische Zwecke errichteten Schutzhütten in ein komplexes soziales Gefüge einordneten. Dieses Gefüge veranschaulicht der Reisebericht aus dem Jahre 1897 von Karl Kollbach, einem Alpinisten aus dem Deutschen Reich, über seine Wanderungen in der Hohen Tatra. Unterwegs mit einem Führer aus der Region traf er in der verlassenen Berglandschaft auf eine Familie mit einer Rinderherde, die in einer einfachen Hütte hauste; auch in der Roztoka-Schutzhütte (Schronisko w Dolinie Roztoki) der Tatra-Gesellschaft lebten drei polnische Jugendliche den Sommer über und ein in der Nähe stationierter Grenzgendarm leistete ihnen öfters Gesellschaft. In der Schutzhütte am Ufer des Meerauges traf Kollbach schließlich auf andere Reisende. Mit einem polnischen Professor, der den ganzen Sommer dort verbrachte, einem österreichischen Soldaten und einem polnischen Gymnasiasten fühlte er sich so sehr verbunden, dass er folgerte, dass ,wenigstens in diesem stillen Bergwinkel die Gegensätze zwischen Deutschtum und Polentum nicht vorhanden" seien. ${ }^{50}$ Welch eine große Rolle das ähnliche soziale Milieu der Reisenden als verbindendes Element entgegen nationaler Differenzen spielte, zeigt sich bereits wenige Tage später, als er im selben Bericht die großen Unterschiede der deutschen und polnischen Bevölkerung in den oberschlesischen Dörfern hervorhob. Auch die Hütten der Górale wirkten auf ihn wie Häuser „eines weltfernen Volkes“ und die Dörfer auf dem Weg von Krakau nach Zakopane erinnerten ihn an die erst unlängst besuchte Berliner Kolonialausstellung. ${ }^{51}$

Die von Gebirgsvereinen errichteten Schutzhütten unterschieden sich von den Hütten der einfachen Bevölkerung. In einer vom Deutschen und Österreichischen Alpenverein herausgegebenen Broschüre „Über Hüttenbau“ aus dem Jahre 1877 lassen sich einige Empfehlungen finden. Solche Baupläne und Erfahrungswerte aus dem Alpenraum wurden auch von den Mitgliedern der Karpatenvereine rezipiert. ${ }^{52}$ In der Broschüre grenzte der Autor Johann Stüdl, ein Unternehmer und Mitglied des Alpenvereins aus Prag, die Schutzhütte deutlich von Touristenhäusern und Hotels ab. ${ }^{53}$ Für die Errichtung einer Schutzhütte brauche es keinen Architekten und ihre Ausstattung soll einfach gehalten sein: Neben einer Schlafstätte beinhalteten Stüdls Empfehlungen Koch- und Essgeräte, eine Petroleumlampe, eine Apotheke, Kartenspiele und ein Hüttenbuch mit Kasse für die zu entrich-

49 Müller 1896, S. $151 \mathrm{f}$.

50 Kollbach 1897, S. 71 ff., 79 f.

51 Kollbach 1897, S. 59, 62.

52 Siehe: Vereins-Verhandlungen. 1881. In: Jahrbuch des Siebenbürgischen Karpathen-Vereins 1, S. 158.

53 Stüdl 1877, S. 169 f. 


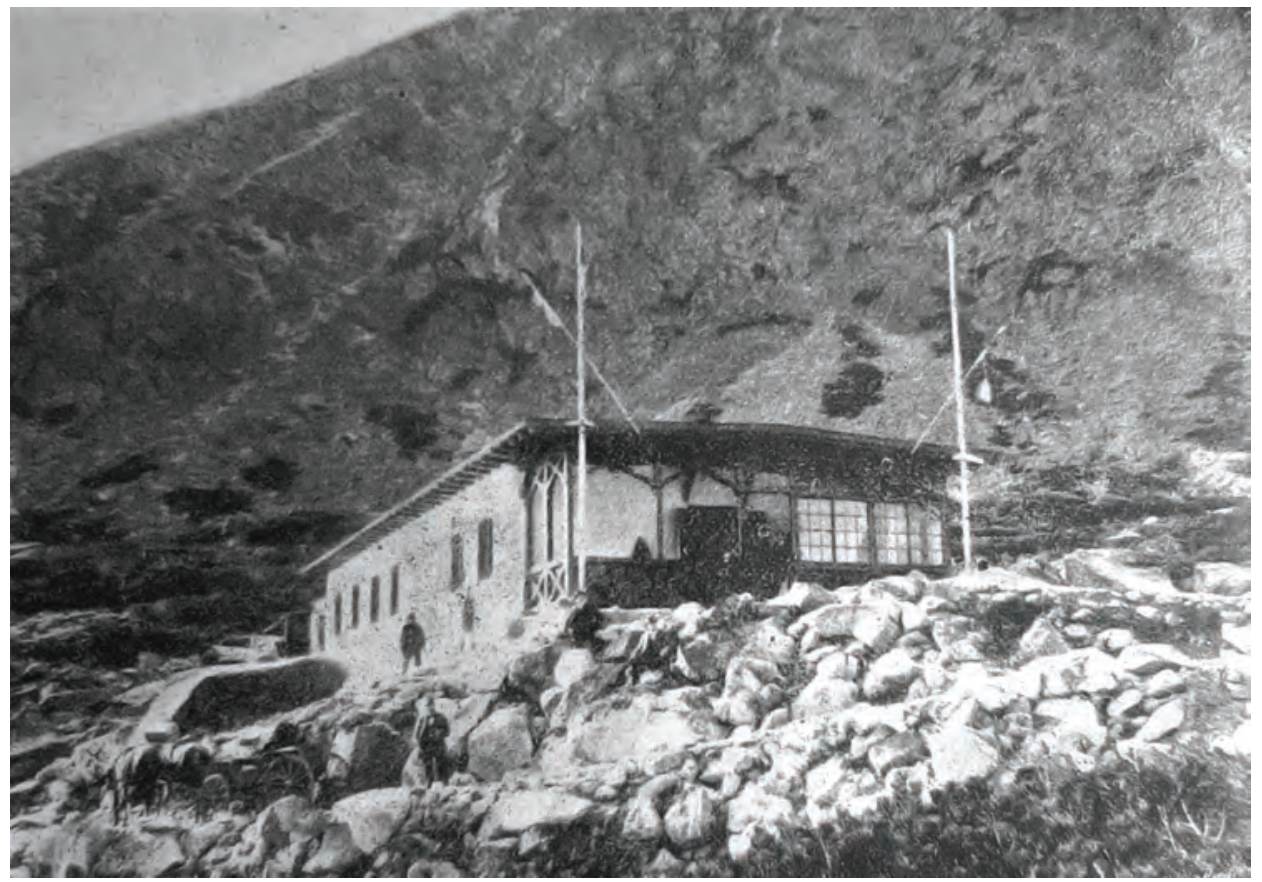

3. Johannes Müller, 1896, Das Schlesierhaus am Felker-See. In: Jahrbuch des Ungarischen Karpathen-Vereines 22, S. 143 (J. Müller, Das Schlesierhaus am Felker-See, „Jahrbuch des Ungarischen Karpathen-Vereines" 22, 1896, s. 143)

tenden Gebühren. ${ }^{54}$ Viele der von Vereinen initiierten Schutzhütten wurden von lokalen Handwerkern, vor allem Zimmerleuten und Maurern, errichtet. ${ }^{55}$ Ausnahmen betreffen vor allem regionale Tourismuszentren, wo unternehmerisch tätige Vereinsmitglieder dieselben Architekten für Schutzhütten engagierten, die bereits Gasthäuser und Sehenswürdigkeiten in der Region entworfen hatten. So hatte beispielsweise der Architekt Gedeon Majunke aus Georgenberg/Špišská Sobota/ Szepesszombat für mehrere Häuser um den Kurort Altschmecks verantwortlich gezeichnet, bevor er sich 1899 im Auftrag des Ungarischen Karpathenvereins der nach dem ungarischen Arzt Edmund Viliam Téry benannten Tery-Hütte (Téryho chata/Téry menedékház) annahm.

In seinen Ausführungen zum Hüttenbau zeigte sich Stüdl bewusst, dass die Errichtung einer Schutzhütte nicht in einem leeren Raum stattfand und es noch an ,verständnisvollem Entgegenkommen von Seite der betreffenden Thalbewohner" mangelte. ${ }^{56}$ Ähnliche Bemerkungen finden sich in vielen zeitgenössischen Reiseberichten wieder, in denen man von der misstrauischen, verängstigten oder gar belustigten Bergbevölkerung erfährt. Bei ihnen hatten Schutzhütten genauso wie die andere errichtete Infrastruktur einen schweren Stand und die Listen

\footnotetext{
54 Stüdl 1877, S. 183-186.

55 Stüdl 1877, S. 169.

56 Stüdl 1877, S. 191.
} 
der Schutzhütten waren gezeichnet von Wiederaufbau- und Reparaturarbeiten. ${ }^{57}$ Die Jahresberichte der Sektionen dokumentierten den „Vandalismus der Landbewohnerschaft ${ }^{\text {“58 }}$ und beschrieben die Schutzhütte als „ein willkommenes Angriffsobjekt für unser dem Fortschritt abholdes Hirtenvolk". ${ }^{59} \mathrm{Oft}$ wurden Hütten geplündert oder durch Steinschlag, Stürme und menschliche Einwirkung beschädigt. Blitzeinschläge und Unfälle durch Lampen oder den Herd setzten die Bauten wiederholt in Brand..$^{60}$ Vermutete Brandstiftung bot Grund für Unmut und Schuldzuweisungen der Vereinsmitglieder entlang der Konfliktlinien nationaler Zugehörigkeit. ${ }^{61}$

Vor diesem Hintergrund beschäftigten sich die Gebirgsvereine intensiv mit der Frage, wie sie ihre Hütten besser schützen konnten, und unternahmen regelmäßig Erkundungstouren. Nicht selten fanden sie die Schutzhütten in desolatem Zustand vor. Fenster, Mobiliar und anderweitige Ausstattung waren ein beliebtes Diebesgut, weshalb die Inneneinrichtung außerhalb der Saison jeweils ins Tal abtransportiert wurde. Häufiger jedoch wiesen die Hütten Spuren uneingeladener Gäste auf, die sich unrechtmäßig Zugang zur Hütte verschafft hatten, indem sie Fenster und Türen aufbrachen. Mehrere Berichte zeugen davon, dass Hirten mit ihren Herden aus der Umgebung die Schutzhütten genauso in Anspruch nahmen, wie die Reisenden auf die Gastfreundschaft der Hirten und ihre Behausungen zurückgriffen. Dies war daran zu erkennen, dass analog zu den Sennhütten in der Mitte des Raums ein offenes Feuer gezündet wurde und der gesamte Boden des Innenraums mit Mist von der Viehherde bedeckt war. Angesichts der damit verbundenen Kosten für die Säuberung und Reparatur überlegte sich ein Reisender gar, ob man lieber von Schutzhütten auf mobile Zelte umsteigen sollte. ${ }^{62}$

Um die lokale Bevölkerung von Zerstörungen, Diebstahl und unsachgemäßer Nutzung abzuhalten, setzten die Gebirgsvereine auf eine Reihe von Maßnahmen. Wenn die Hütte nicht bewirtet war und vom Hüttenwart bewacht werden konnte, griffen sie auf lokale Autoritäten zurück. Sie baten die Gendarmerie, neben dem Grenzübergang auch ein wachsames Auge auf ihre Hütte zu werfen, und die lokalen Geistlichen, die Hirten über den Wert und Zweck einer Schutzhütte zu belehren. ${ }^{63}$ Diese Maßnahmen zeigten jedoch nur mäßig Erfolg. Auch die Weihung

57 Beispielsweise: Geschichtlicher Überblick über die Schutzhütten des SKV 1881-1943. 1990. In: Heinz Heltmann und Helmut Roth (Hrsg.): Der Siebenbürgische Karpatenverein 1880 1945. Gedenkband. Thaur bei Innsbruck: Wort und Welt Verlag. S. 27 ff.

58 Sektionsberichte. Sektion ,Szittnya'. 1885. In: Jahrbuch des Ungarischen Karpathen-Vereines 12, S. 227.

59 Jahresberichte der Sektionen unseres Vereines. Sektion ,Hermannstadt'. 1897. In: Jahrbuch des Siebenbürgischen Karpathen-Vereins 17, S. 97.

${ }^{60}$ Eine solche Episode wird geschildert in: Jahresberichte der Sektionen unseres Vereines. Sektion ,Kronstadt'. 1898. In: Jahrbuch des Siebenbürgischen Karpathen-Vereins 18, S. 111.

${ }^{61}$ Pelc 2014, S. 237 f.; Hoenig 2018, S. 51.

62 Poschner 1887, S. 140.

63 Jahresberichte der Sektionen unseres Vereines. Sektion Bistritz-Naszod-Rodna. 1884. In: Jahrbuch des Siebenbürgischen Karpathen-Vereins 4, S. 166. 
des Baus durch den Pfarrer in Gegenwart der lokalen Bevölkerung, das Aufstellen eines Kreuzes vor der Hütte und das Anbringen von heiligen Bildern im Innern bewahrten die Schutzhütten nicht vor Zerstörungen. ${ }^{64}$ Weitaus erfolgreicher waren dafür die Christbescherungen zu Weihnachten, wie sie nach dem Vorbild der Alpenvereine veranstaltet wurden, um bei der ärmlichen Dorfjugend Sympathie für das Anliegen der Gebirgsvereine zu erwecken. ${ }^{65}$ Erst mit fortschreitendem Ausbau der Infrastruktur und der größeren Anzahl Reisenden im Gebirgsraum wurde es jedoch einfacher für die Vereine, Zerstörungen und unerlaubte Nachtlager in ihren Hütten zu verhindern.

\section{Zusammenfassung: Idealisierung als aktive Raumaneignung}

Das Ziel des Artikels bestand darin, zu zeigen, wie der Hüttenbau als Pionierprojekt in der touristischen Erschließung von Gebirgsräumen funktionierte. Die Schutzhütten waren Teil einer Reihe von Maßnahmen, um den Gebirgsraum für Reisende schrittweise komfortabler zu gestalten. Durch den Einbezug lokaler Fabrikanten und Handwerker betteten sich die Hütten in die allgemeine Bewerbung regionaler Produkte ein. ${ }^{66}$ Die Reisenden wurden zudem von lokalen Bergführern begleitet, die teilweise in Tracht auftraten und damit für die nötige Bergromantik sorgten.$^{67}$ Mit der Zeit machte die einfache Infrastruktur der Schutzhütte häufig einem größeren Restaurant oder einer Hotelanlage Platz. Wenn sie dabei nicht ersetzt wurden, führte dies zu einer Umnutzung der Schutzhütte. Ein solches Beispiel ist die erste Schutzhütte in der Tatra, die Rainer-Hütte im Kohlbachtal, die dem neuen Hotel Gemse (Kamzík) nach dessen Bau im Jahre 1884 als Holzstall und Weinkeller diente (Bild 4). Eine Rekonstruktion der damaligen, 1863 auf Initiative des Gastwirts Johann Georg Rainer erbauten Schutzhütte bietet heute als Museum des Tatra-Nationalparks einen Einblick in den Alpinismus des 19. Jahrhunderts.

Indem die Schutzhütte Vorreiter einer touristischen Infrastruktur war, wird deutlich, wie sehr sie von der Raumaneignung einer als wild empfundenen Landschaft abhing. Die Hütte befand sich in einem infrastrukturell nicht erschlossenen und deshalb als unbewohnt betrachteten Raum, der im Gegensatz zu den baulichen Ensembles von Dörfern oder gar Städten stand. So wurde in vielen Berichten die Einsamkeit der Schutzhütte in der Gebirgslandschaft hervorgehoben, die in

64 Jahresberichte der Sektionen unseres Vereines. Sektion ,Schielthal'. 1893. In: Jahrbuch des Siebenbürgischen Karpathen-Vereins 13, S. 120.

65 Jahresberichte der Sektionen unseres Vereines. Sektion ,Hermannstadt'. 1897. In: Jahrbuch des Siebenbürgischen Karpathen-Vereins 17, S. 97.

${ }^{66}$ Beispielsweise wurden beim Schlesierhaus des Ungarischen Karpathenvereins für das Mobiliar und Inventar ausdrücklich nur Fabrikanten und Handwerker aus Zips berücksichtigt. Müller 1896, S. 148.

${ }^{67}$ Klipp 2006, S. 129. 


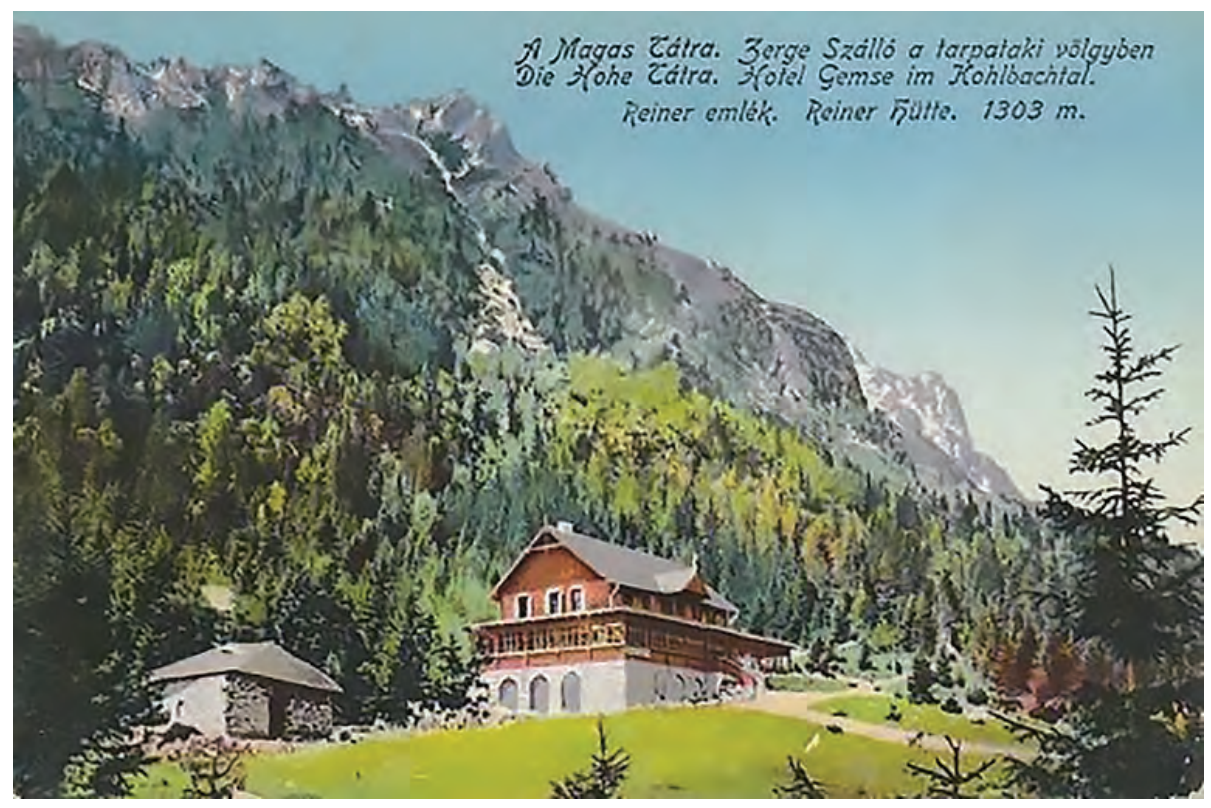

4. Postkarte „Die Hohe Tátra. Hotel Gemse im Kohlbachtal. Reiner Hütte. 1303 m.“, 1913 (Pocztówka „Tatry Wysokie. Hotel Gemse w dolinie Kohlbach. Chata Reinera. 1303 m”, 1913 rok), Sammlung der Autorin (Zbiory autorki)

der Weite für sich alleine stand und sich organisch in die natürliche Umgebung einfügte. Die Hütte diente den Erzählungen als Rahmen, um aus dem Fenster oder vor der Tür bei einer Rast ein Panorama zu betrachten, oder sie wurde selbst zum Teil der Bergszenerie, wie dies auf der bereits erwähnten Lithographie von Kořistka der Fall war. Diese Abgeschiedenheit der Schutzhütte blieb auch dann Teil der touristischen Erwartungshaltung, wenn sie bereits mit einer Hotelanlage ersetzt werden sollte. Ernst Lüdecke, der 1883 einer Sondierungskommission des SVK für eine neue Schutzhütte am Bulea-See (Lacul Bâlea) angehörte, beschrieb treffend das Spannungsverhältnis, in dem sich viele Mitglieder von Gebirgsvereinen in der touristischen Erschließung der Berge wiederfanden. Obwohl ihr Ziel die Ermöglichung selbständiger Touren durch Veränderung der Landschaft war, fürchteten sie diese gleichzeitig: „Gibt es doch noch so Manchen, der da in allem Ernste wünscht: Es möge die in der Ebene fortschreitende Kultur ihre Fittige niemals in unsere Berge lenken, damit bequeme Hotels mit süsslächelnden Wirthen und befrackten Kellnern diese naturwüchsige Romantik nicht verscheuchen" ". ${ }^{6}$

In dieser Aussage widerspiegelt sich die Funktion der Schutzhütte, die sie in den meisten Reiseberichten einnimmt und dessen soziale Dimension dieser Artikel hervorgehoben hat. Sie war Ausgangs- und Endpunkt der Reise und bot der eigentlichen Unternehmung eine Bühne, indem sie die Beschreibungen der Strapazen der Wanderung und der wunderbaren Ausblicke rahmte. Die Schutz-

${ }^{68}$ Lüdecke 1884, S. 101. 
hütte bot den Reisenden die Möglichkeit, die Berge selber zu idealisieren, indem sie den Gebirgsraum durch die eigene Raumaneignung aktiv mitgestalteten. Die Einbettung der Hütte in die Landschaft formte die soziale Ordnung in der Region neu und zeigt deshalb, wie zentral die Nutzung der Berge für den Prozess ihrer Idealisierung ist.

\section{Literatur}

Barcza, Emerich (1904): In den Siebenbürgischen Karpaten. In: Jahrbuch des Ungarischen Karpathenvereins 31, S. 75-94.

Brunnbauer, Ulf / Robert, Pichler (2002): Mountains as "lieux de mémoire”. Highland Values and Nation-Building in the Balkans. In: Balkanologie 6.1-2, S. 77-100.

Cosgrove, Denis (2006), Modernity, Community and the Landscape Idea. In: Journal of Material Culture 11(1/2), S. 49-66

Crowley, David (2001), Finding Poland in the Margins: the Case of the Zakopane Style. In: Journal of Design History 14.2, S. 105-116.

Dabrowski, Patrice (2005), "Discovering” the Galician Borderlands: The Case of the Eastern Carpathians. In: Slavic Review 64.2, S. 380-402.

Dabrowski, Patrice (2008), Constructing a Polish Landscape: The Example of the Carpathian Frontier. In: Austrian History Yearbook 39, S. 45-65.

Dénes, Franz (1888): Wegweiser durch die Ungarischen Karpaten. Leutschau: Verlag des Ungarischen Karpathenvereins.

Eljasz Radzikowski, Walery (1903) Wspomnienie o schroniskach nad Morskim Okiem. In: Pamiętnik Towarzystwa Tatrzańskiego 24, S. 120-132.

Geschichtlicher Überblick über die Schutzhütten des SKV 1881-1943. 1990. In: Heltmann, Heinz I Roth, Helmut (Hrsg.): Der Siebenbürgische Karpatenverein 1880-1945. Gedenkband. Thaur bei Innsbruck: Wort und Welt Verlag, S. 27-29.

Grupp, Peter (2003): Faszination Berg: die Geschichte des Alpinismus. Köln-Weimar-Wien: Böhlau, S. $172-181$.

Heksch, Alexander F. (1885): Illustrirter Führer durch Pressburg und seine Umgebungen, das Waagthal und die kleinen Karpathen. Wien-Pressburg: A. F. Heksch's Verlag.

Heltmann, Heinz (1990): Zur Geschichte des Siebenbürgischen Karpatenvereins. In: Heltmann, Heinz/Roth, Helmut (Hrsg.): Der Siebenbürgische Karpatenverein 1880-1945. Gedenkband. Thaur bei Innsbruck: Wort und Welt Verlag, S. 11-26.

Heppner, Harald (Hrsg.) (2015): Die Erschließung der Karpaten. Themenheft. Danubiana Carpathica 8.

Hoenig, Bianca (2014): Kleinod und Ressource: Die Polnische Naturschutzbewegung und die Tatra. In: Bohemia 54.1, S. 56-73.

Hoenig, Bianca (2018). Geteilte Berge. Eine Konfliktgeschichte der Naturnutzung in der Tatra. Göttingen: Vandenhoeck \& Ruprecht.

Jahresberichte der Sektionen unseres Vereines. Sektion Broos. 1884. In: Jahrbuch des Siebenbürgischen Karpathen-Vereins 4, S. 146-151.

Jahresberichte der Sektionen unseres Vereines. Sektion Bistritz-Naszod-Rodna. 1884. In: Jahrbuch des Siebenbürgischen Karpathen-Vereins 4, S. 165-167.

Jahresberichte der Sektionen unseres Vereins. Sektion „Hermannstadt“. 1886. In: Jahrbuch des Siebenbürgischen Karpathen-Vereins 6, S. 199-207.

Jahresberichte der Sektionen unseres Vereines. Sektion „Hermannstadt“. 1897. In: Jahrbuch des Siebenbürgischen Karpathen-Vereins 17, S. 91-101. 
Jahresberichte der Sektionen unseres Vereines. Sektion „Kronstadt“. 1898. In: Jahrbuch des Siebenbürgischen Karpathen-Vereins 18, S. 110-116.

Jahresberichte der Sektionen unseres Vereines. Sektion „Schielthal“. 1893. In: Jahrbuch des Siebenbürgischen Karpathen-Vereins 13, S. 118-121.

Judson, Pieter M. (2014): Reisebeschreibungen in der "Südmark“ und die Idee der deutschen Diaspora nach 1918. In: Stachel, Peter / Thomsen, Martina (Hgs.): Zwischen Exotik und Vertrautem. Zum Tourismus in der Habsburgermonarchie und ihren Nachfolgestaaten. Bielefeld: transcript, S. 59-76.

Kalchbrenner, Károly (1875): Ein Ausflug in das Kupferschächtenthal. In: Jahrbuch des Ungarischen Karpathen-Vereines 2, S. 219-245.

Kerschbaumer, Gabriel (2017): Hochalpine Architektur. Wien: Klein Publishing.

Keym, Stefan (2008): Kulturhauptstadt des geteilten Polen? Zum Beitrag der Musik zur Stilisierung Zakopanes und der Tatra-Region zu einem nationalen Wertezentrum. In: Altenburg, Detlef / Ehrlich, Lothar / John, Jürgen (Hrsg.): Im Herzen Europas. Nationale Identitäten und Erinnerungskulturen. Köln-Weimar-Wien: Böhlau, S. 307-325.

Klipp, Anton (2006): Die Hohe Tatra und der Karpathenverein. Karlsruhe: Karpathendeutsches Kulturwerk Slowakei e.V.

Kollbach, Karl (1897): Wanderungen durch die deutschen Gebirge. Von der Tatra bis zur Sächsischen Schweiz. Eine Wanderung durch die Karpathen, Beskiden, das Altvater-, Glatzer, Riesen-, Böhmische Mittelgebirge und die Sächsische Schweiz. Köln: Paul Aeubner.

Kořistka, Carl (1864): Die Hohe Tatra in den Central-Karpaten. Eine geographische Skizze verfasst auf Grundlage einer Bereisung. Ergänzungsheft No. 12 zu Petermann’s „Geographischen Mittheilungen “. Gotha: Justus Perthes.

Kulig, Marzena (2003): Architektura tatrzańskich schronisk górskich Polskiego Towarzystwa Tatrzańskiego $w$ dwudziestoleciu międzywojennym. Warszawa: Neriton.

Kurek, Artur / Wójcik, Wiesław A. (Hrsg.) (2016): Z dziejów Towarzystwa Tatrzańskiego i Polskiego Towarzystwa Tatrzańskiego (1873-1950). Kraków: Oficyna Wydawnicza „Wierchy”.

L. (1898): Das „Friedrich“-Schutzhaus. In: Jahrbuch des Ungarischen Karpathen-Vereines 25, S. $156-168$.

Lipták, L‘ubomír (2003): Die Tatra im slowakischen Bewusstsein. In: Stekl, Hannes / Mannová, Elena (Hrsg.): Heroen, Mythen, Identitäten. Die Slowakei und Österreich im Vergleich. Wien: WUV-Universitätsverlag, S. 261-288.

Lövy, Emma (1910): Ein Ausflug in die Rodnaer Alpen. In: Jahrbuch des Ungarischen KarpathenVereines 37, S. 16-40.

Lüdecke, Ernst (1884): Ein Ausflug zum Bullea See (29. Juni bis 1. Juli 1883). In: Jahrbuch des Siebenbürgischen Karpathen-Vereins 4, S. 96-108.

MacFarlane, Robert (2003): Mountains of the Mind. New York: Pantheon Books.

Magnesius, Hermann (1897): Ein Ausflug auf Schneeschuhen in das Zibinsgebirge. In: Jahrbuch des Siebenbürgischen Karpathen-Vereins 17, S. 72-78.

Malzner, Sonja / Peiter, Anne D. (Hrsg.) (2018): Der Träger. Zu einer „tragenden“ Figur der Kolonialgeschichte. Bielefeld: transcript.

Matuska, Thomas (2012): Die Erforschung und alpinistische Erschließung der Karpaten. In: Das achtzehnte Jahrhundert und Österreich: Jahrbuch der Österreichischen Gesellschaft zur Erforschung des Achtzehnten Jahrhunderts 26, S. 255-265.

Mitchell, W. J. T. (2002): Landscape and Power. Chicago: University of Chicago Press.

Morin, Karen M. (2009): Landscape: Representing and Interpreting the World. In: Clifford, Nicholas J. / Holloway, Sarah L. / Rice, Stephen P. / Valentine, Gill (Hrsg.): Key Concepts in Geography. Los Angeles et al.: Sage, S. 286-299.

Müller, Johannes (1896): Schlesierhaus am Felker-See. In: Jahrbuch des Ungarischen Karpathenvereins $23, \mathrm{~S} .142-154$.

Góry - Literatura - Kultura 13, 2019

(C) for this edition by CNS 
Pelc, Martin (2008): Pohorská jednota „Radhošt'“ a Klub českých turistů: problém vztahu regionu. In: Acta Historica Universitatis Silesianae Opaviensis. Confinia Silesiae 1, S. 425-437.

Pelc, Martin (2014): Orte der Selbstpositionierung. Deutsche und tschechische Wandervereine vor 1945. In: Stachel, Peter / Thomsen, Martina (Hrsg.): Zwischen Exotik und Vertrautem. Zum Tourismus in der Habsburgermonarchie und ihren Nachfolgestaaten. Bielefeld: transcript, S. 233-242.

Polášková, Jiřina / Polášek, Jaromír (2009): Historie Beskydské turistiky. Sto dvacet let organizované turistiky v Moravskoslezských Beskydách. Turistické chaty na Frýdecko-Misteku, Třinecku a Jablunovsku. Ostrava: Šmírna-Print.

Poschner, G. (1887): Einige Exkursionspunkte der Sektion Bistritz-Nassod-Rodna des siebenbürgischen Karpathenvereines. In: Jahrbuch des Siebenbürgischen Karpathen-Vereins 7, S. 117-148.

Posewitz, Theodor (1894): Die Geschichte des Ungarischen Karpathen-Vereines seit seinem Bestande. In: Jahrbuch des Ungarischen Karpathen-Vereines 21, S. 1-211.

Pratt, Marie Louise (1992): Imperial Eyes. Travel Writing and Transculturation. London-New York: Routledge.

Rey, Mieczysław (1876): Zagajenie pierwszego walnego zgromadzenia Towarzystwa Tatrzańskiego. In: Pamiętnik Towarzystwa Tatrzańskiego 1, S. 7-8.

Scharfe, Martin (2007): Berg-Sucht: eine Kulturgeschichte des frühen Alpinismus 1750-1850. WienKöln-Weimar: Böhlau.

Scharr, Kurt (Hrsg.) (2004): Die Karpaten. Balthasar Hacquet und das „vergessene“ Gebirge in Europa. Innsbruck: Studienverlag.

Schroniska i altany Tow. Tatrz. w Tatrach. 1914. In: Pamiętnik Towarzystwa Tatrzańskiego 35, S. LXVI-LXVII.

Schüler, Jaroslav (1937): Weekendové chaty. Praha: J. Sovček.

Sektionsberichte. Sektion „Szittnya“. 1885. In: Jahrbuch des Ungarischen Karpathen-Vereines 12, S. 227-229.

Stephen, Leslie (1871): The Playground of Europe. London: Longmans Green.

Der Streit um das Meerauge. 1909. In: Österreichische Zeitschrift für Vermessungswesen 7.9, S. 279-281.

Stüdl, Johann [1877]: Ueber Hüttenbau. In: Zeitschrift des Deutschen und Osterreichischen, Alpenvereins 8, S. 169-191.

Toth, Adalbert (1990): Die Genehmigungspraxis politischer Vereine und Parteien in Ungarn 1892-1896. In: Ungarn-Jahrbuch 18, S. 75-105.

Vereins-Verhandlungen. 1881. In: Jahrbuch des Siebenbürgischen Karpathen-Vereins 1, S. 153-169.

Volker, Matthies (2018): Im Schatten der Entdecker. Indigene Begleiter europäischer Forschungsreisender. Berlin: Christoph Links Verlag.

Ważniejsze pisma wystosowane do Towarzystwa Tatrzańskiego. 1876. In: Pamiętnik Towarzystwa Tatrzańskiego 1, S. 36-44.

Weber, Samuel (1897): Franz Klein, das Koscielisker Thal und der Grosse Fisch-See. In: Jahrbuch des Ungarischen Karpathen-Vereines 24, S. 34-45.

Wedekind, Michael (2004): Der Siebenbürgische Karpatenverein (1880-1944). Ein Beitrag zur Sozialgeschichte Siebenbürgens. In: Halbjahresschrift für südosteuropäische Geschichte, Literatur und Politik 16.2, S. 71-86.

Zimmer, Oliver (1998): In Search of Natural Identity: Alpine Landscape and the Reconstruction of the Swiss Nation. In: Comparative Studies in Society and History 40.4, S. 637-665.

Góry - Literatura - Kultura 13, 2019

(C) for this edition by CNS 